\title{
Performance of $k-\omega$ and $k-\varepsilon$ Model for Blood Flow Simulation in Stenosed Artery
}

\author{
Md. Alamgir Kabir ${ }^{*}$, Kausari Sultana, and Md. Ashraf Uddin \\ Department of Mathematics, Shahjalal University of Science and Technology, Sylhet-3114, Bangladesh
}

\begin{abstract}
Blood flow through arterial stenosis can play a crucial role at the post stenotic flow regions. This produces a disturbance in the normal flow path. The intensity of the flow disturbance (i.e. laminar, transitional and turbulent flow characteristics) depends not only on the severity of the stenosis but also on the pattern of the geometrical model. In that case, the turbulence model plays vital role to measure these flow disturbances. However, it is very important to choose a proper flow simulation model that can predict the flow behavior of fluid accurately and efficiently with less computational cost and time. Thus, this study aims to analyze the results of two turbulence models i.e. $k-\omega$ and $k-\varepsilon$ for blood flow simulation to compare their performance for the prediction of the flow behavior. Simulations have been performed with $75 \%$ area reductions in the arteries. The results of simulation show that, the flow parameters obtained from the $k-\varepsilon$ model exhibits lack of fits with the experimental data. On the other hand, $k-\omega$ model can capture large scale gradient in the different parameters of blood flow and has a good agreement with the experimental data. This study suggests that, $k$ - $\omega$ model has the better performance comparing to $k-\varepsilon$ model to predict the behavior of blood flow in stenosed artery.
\end{abstract}

(C) 2020 Published by Bangladesh Mathematical Society

Received: August 11, $2020 \quad$ Accepted: November 23, $2020 \quad$ Published Online: January 15, 2021

Keywords: Stenosis; Turbulence; Artery; Wall shear stress

AMS Subject Classifications 2020: 76F02; 76F10; 76Z99

\section{Introduction}

The accumulation of cholesterols and the propagation of connective tissues for a longer period in an arterial wall initiate the formation of plaques. Over time, these plaques build up in the walls of arteries and gradually limit the normal blood flow. Once this constriction of the arterial tube becomes large enough and occludes the blood flow, it produces flow separations and flow circulation throughout the post stenotic zone. These disturbances of the flow pattern may provoke the further rapid growth of the stenosis towards the downstream regions.

The turbulent flow at the post stenotic region has a great influence to represent an abnormal flow condition especially for flow circulation and flow separation towards the tube. This abnormal flow is strongly related to various vascular disorders such as post-stenotic dilatation $[1,2]$ which has an important clinical consequence. In addition, high pressure drops and high wall shear stresses at the throat of the stenosis have significant effects on damaging blood cell materials and can activate the blood platelets [3]. Thus, from the pathological point of view, there is a considerable interest among the researchers to gain a better insight into the dynamics of post-stenotic blood flows. Thus, numerous experimental studies have been performed on laboratory models [1,4] including animals and human [4-9].

\footnotetext{
* Corresponding Author.Email Address:akabir-mat@gmail.edu
} 
To get details flow patterns, different noninvasive numerical methods have been offered and at present computational fluid dynamics (CFD) technique is considered as one of the most popular tools for gaining accurate results and visualizing the flow field properly. In most of the cases, the numerical modeling in blood flow, particularly in stenotic flow are limited in laminar flow model with low Reynolds numbers [10-12]. However, another study [13] used $k-\varepsilon$ turbulence model for Reynolds numbers between 2500 and 194,000 to predict turbulent characteristics in stenotic artery. But the result is unrealistic in some extent considering the fact that, the normal range of Reynolds number in human circulatory system varies from 1 in small arterioles to approximately 4000 in the largest artery [14]. Moreover, Ghalichi et al. [3] in their investigation used the $k-\omega$ turbulent model in the same stenosed artery that was used by Ahmed and Giddens [4] and observed that $k-\omega$ model works better than standard $k-\varepsilon$ model in some extent. Recently Lee et al. [15] used some axisymmetric stenosed arteries and observed the turbulent flow through them. Their results again suggest that $k$ - $\omega$ model is a handy tool for predicting laminar as well as turbulent flow in the stenosed vessel.

A large number of models are available to predict the behavior of blood flow. In case of numerical model, it is very important to get the accurate results. The model which can predict the results more accurately should be considered. Therefore, considering the above discussions, we have checked the sensitivity of the two equations turbulent models, especially $k-\omega$ and $k-\varepsilon$ models in stenosed artery with $75 \%$ area reduction by mean of computational fluid dynamics technique to predict the turbulence characteristics of blood flow which can play a significant role in diagnosed arterial diseases. In this paper, a comparative analysis has been made between the $k-\omega$ and $k-\varepsilon$ models by simulating the blood flow behaviors and the model showing the best performance have been chosen.

\section{Method}

\subsection{Model Geometry}
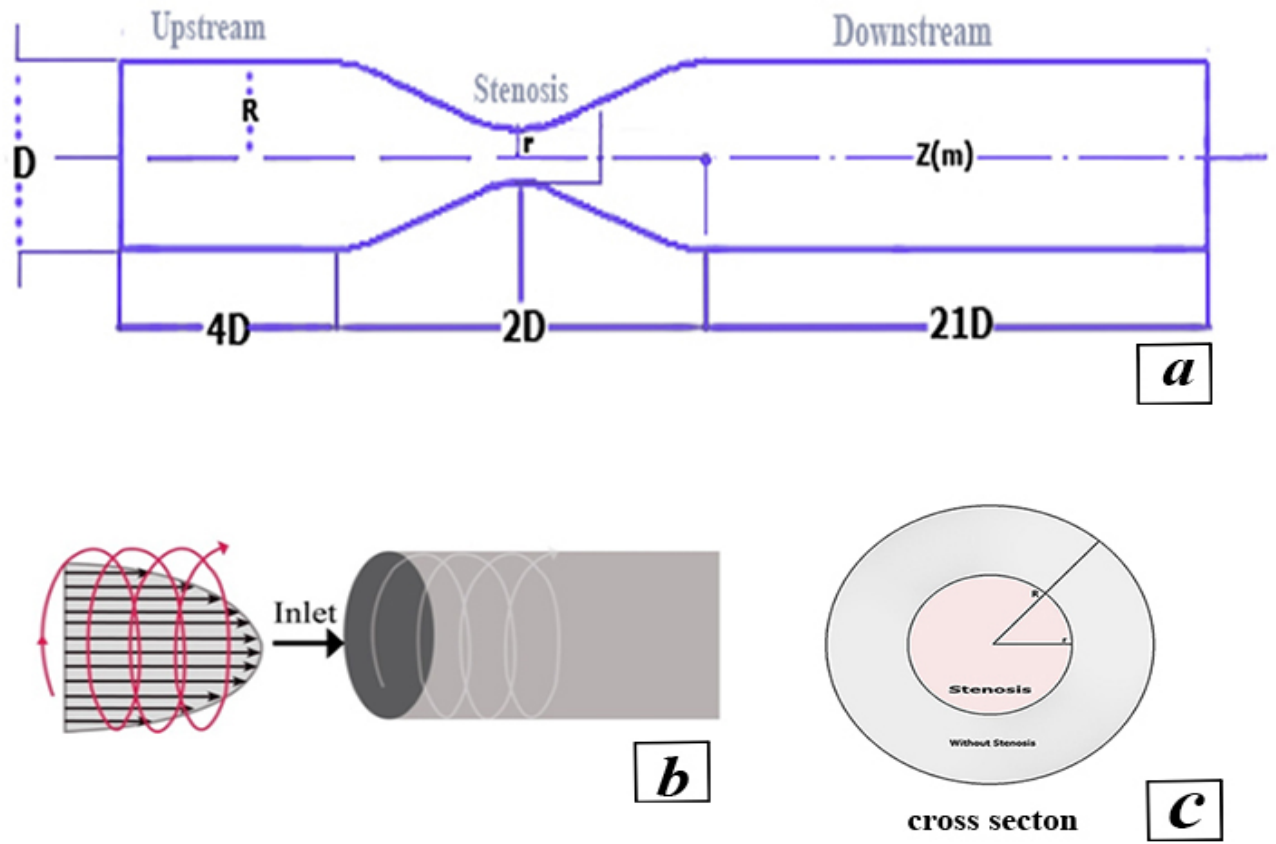

Fig. 2.1. (a) Model stenosed artery, (b) inlet velocity profile and (c) cross-sectional area of the artery.

Model geometry of the stenosed blood vessel has been created by the cosine curve using the following equation:

$$
\frac{r(Z)}{R}=1-\delta_{c}\left[1+\cos \left(\frac{Z \pi}{D}\right)\right],-D \leq Z \leq D
$$


The constrictions of the artery followed in the cosine curve with $75 \%$ area reduction and the parameter $\delta_{c}$ controls the percentage the stenosis. The model artery employed in theoretical calculations by Smith [16] gives almost correct representation of the biological form of arterial stenosis. The total length of the model is considered as $540 \mathrm{~mm}(27 \mathrm{D})$, where the diameter of the model is taken $\mathrm{D}=20 \mathrm{~mm}$ and the length of the upstream region, downstream region and stenosed region are taken as 4D, 21D and 2D, respectively The schematic model geometry is shown in Fig. 2.1.

\subsection{Mathematical Procedure}

The Reynolds averaged Navier-Stokes (RANS) equation is taken as the governing equation for the current study. The Navier-Stokes equations are treated as the governing equations for the motion of blood flow. After the application of Reynolds time-averaging techniques, the Reynolds averaged Navier-Stokes (RANS) are found in tensor form, given as follows:

$$
\begin{aligned}
& \frac{\partial u_{i}}{\partial x_{i}}=0 \\
& \frac{\partial}{\partial t}\left(\rho u_{i}\right)+\frac{\partial}{\partial x_{j}}\left(\rho u_{i} u_{j}\right)=-\frac{\partial p}{\partial x_{i}}+\frac{\partial}{\partial x_{j}}\left[\mu\left(\frac{\partial u_{i}}{\partial x_{j}}+\frac{\partial u_{j}}{\partial x_{i}}\right)\right]+\frac{\partial \tau_{i j}}{\partial x_{j}}
\end{aligned}
$$

where, $x_{i}=(x, y, z)$ are the Cartesian co-ordinate systems, $u_{i}$ are the mean velocity components, $\rho$ is the density, $p$ is the pressure and $\tau_{i j}$ is the Reynolds stress.

Boussinesq hypothesis is used to model the Reynolds stress and the turbulent eddy viscosity is found by using two equations turbulence model such as $k$ - $\omega$ and $k-\varepsilon$ models. Boussinesq hypothesis is used to model the Reynolds stress $\tau_{i j}$ which is given as follows:

$$
\tau_{i j}=-\rho\left\langle u_{i}^{\prime} u_{j}^{\prime}\right\rangle=\mu_{t}\left(\frac{\partial u_{i}}{\partial x_{j}}+\frac{\partial u_{j}}{\partial x_{i}}\right)-\frac{2}{3} \rho k \delta_{i j}
$$

where, $u_{i}^{\prime}$ is the fluctuating velocity components, $k=\frac{1}{2}\left\langle u_{i}^{\prime} u_{j}^{\prime}\right\rangle$ is the turbulent kinetic energy and $\mu_{t}$ is the turbulent eddy viscosity obtained by using the standard $k$ - $\omega$ model of Wilcox. The prime objective of Boussinesq hypothesis is to create relation between the Reynolds stresses to the mean velocity gradients. In the $k$ - $\omega$ model, the eddy-viscosity is modelled as:

$$
\mu_{t}=\frac{\rho k}{\omega}
$$

where, $\omega$ is the specific dissipation rate which can also be considered as the ratio of dissipation rate $(\varepsilon)$ to turbulence kinetic energy $(k)$.

Here $k$ and $\omega$ are obtained from the following equations:

$$
\begin{aligned}
& \frac{\partial k}{\partial t}+\frac{\partial k\left\langle u_{j}\right\rangle}{\partial x_{j}}=-\frac{1}{\rho}\left\langle\rho u_{i}^{\prime} u_{j}^{\prime}\right\rangle \frac{\partial\left\langle u_{i}\right\rangle}{\partial x_{j}}-\beta^{*} k \omega+\frac{\partial}{\partial x_{j}}\left[\frac{1}{\rho}\left(\mu+\sigma^{*} \mu_{t}\right) \frac{\partial k}{\partial x_{j}}\right] \\
& \frac{\partial \omega}{\partial t}+\frac{\partial \omega\left\langle u_{j}\right\rangle}{\partial x_{j}}=-\alpha_{1} \frac{\omega}{\rho k}\left\langle\rho u_{i}^{\prime} u_{j}^{\prime}\right\rangle \frac{\partial\left\langle u_{i}\right\rangle}{\partial x_{j}}-\beta \omega^{2}+\frac{\partial}{\partial x_{j}}\left[\frac{1}{\rho}\left(\mu+\sigma \mu_{t}\right) \frac{\partial \omega}{\partial x_{j}}\right]
\end{aligned}
$$

where, $\sigma^{*}=0.5, \beta^{*}=0.072, \sigma=0.5, \alpha_{1}=1.0, \beta=0.072$

Again, the turbulence kinetic energy, $k$ and its dissipation rate, $\varepsilon$ are obtained from the following transport equations:

$$
\frac{\partial(\rho k)}{\partial t}+\frac{\partial}{\partial x_{j}}\left(\rho k u_{i}\right)=\frac{\partial}{\partial x_{j}}\left[\frac{\mu_{t}}{\sigma_{k}} \frac{\partial k}{\partial x_{j}}\right]+2 \mu_{t} S_{i j} S_{i j}-\rho \varepsilon
$$




$$
\frac{\partial(\rho \varepsilon)}{\partial t}+\frac{\partial}{\partial x_{j}}\left(\rho \varepsilon u_{i}\right)=\frac{\partial}{\partial x_{j}}\left[\frac{\mu_{t}}{\sigma_{\varepsilon}} \frac{\partial \varepsilon}{\partial x_{j}}\right]+C_{1 \varepsilon} \frac{\varepsilon}{k} 2 \mu_{t} S_{i j} S_{i j}-2 C_{2 \varepsilon} \rho \frac{\varepsilon^{2}}{k}
$$

where, turbulent eddy viscosity is obtained from the relation,

$$
\mu_{t}=\rho c_{\mu} \frac{k^{2}}{\varepsilon}
$$

The generation of TKE due to the mean velocity gradients is represented by $G_{k}$ and can be defined as the following manner which is somewhat similar like Boussinesq hypothesis,

$$
G_{k}=\mu_{t} S^{2}
$$

Here, $S$ represent the modulus of the mean rate-of-strain tensor and is defined as,

$$
S=\sqrt{2 S_{i j} S_{i j}}
$$

where $\mathrm{c}_{\mu}=0.09, \sigma_{\mathrm{k}}=1.0, \sigma_{\varepsilon}=1.3, \mathrm{C}_{1 \varepsilon}=1.44$ and $\mathrm{C}_{2 \varepsilon}=1.92$ are adjustale constants.

\subsection{Non-Newtonian Model}

As blood in human body contains two parts such as liquid and solid parts and the liquid part of the blood, which is called plasma, generally made of water, salts, and protein and over $50 \%$ of blood in human body is plasma. That is why, blood is considered as incompressible and Newtonian-homogeneous fluid with a density $(\rho)$ of $1060 \mathrm{~kg} / \mathrm{m}^{3}$ and a constant dynamic viscosity $(\mu)$ of $3.71 \times 10-3 \mathrm{~Pa}$ s. But, in microcirculatory system such as small branches and capillaries, blood exhibits nonNewtonian phenomenon and in this study blood is treated as Non-Newtonian fluid. Here, the well-known Carreau [15] model has been used to capture the non-Newtonian behavior of the fluid and the model is defined as:

$$
\mu(|\dot{\gamma}|)=\mu_{\infty}+\left(\mu_{0}-\mu_{\infty}\right)\left[1+(\lambda \dot{\gamma})^{2}\right]^{n-1 / 2}
$$

where, $\mu_{\infty}=0.00345 \mathrm{~Pa} \mathrm{~s}$ is the infinite shear viscosity, $\mu_{0}=0.056 \mathrm{~Pa} \mathrm{~s}$ is the blood viscosity at zero shear rate, $\gamma$ is the instantaneous shear rate, $\lambda=3.313 \mathrm{~s}$ is the time constant which is associated with the viscosity that changes with shear rate and $n=0.3568$ is the power-law index.

\subsection{Boundary Conditions}

The geometry of the existing model is considered as nonflexible and solid circular tube. The inlet boundary condition plays a crucial role to study in blood flow through arterial stenosis. A parabolic velocity profile has been applied at the inlet of the model. No-slip boundary condition is used at the artery wall. Moreover, the model outlet has been treated as a pressure outlet using the default setting.

The parabolic velocity profile is computed by the equation,

$$
v(x, y)=2 V\left[1-\left(\frac{r}{R}\right)^{2}\right]
$$

Here, $\mathrm{V}$ is the bulk stream-wise velocity related to the Reynolds number, $\mathrm{Re}=\rho \mathrm{VD} / \mu$ of the blood flow

\subsection{Computational Procedure}

With a view to performing numerical solution for current study, commercially available Computational Fluid Dynamics (CFD) software, Fluent has been used. Fluent uses finite volume method (FVM) for discretization of flow governing equations. The finite volume method evaluates partial differential equations in algebraic equations form by an iterative process. Pressure based solver has been applied to solve the flow equations with implicit formulation method. Also the Semi-Implicit Method for Pressure-Linked Equation (SIMPLE) scheme of [17] pressure-velocity coupling has been 
used. For discretization process, second order upwind scheme has been used for the equations of turbulent kinetic energy $(k)$, momentum, dissipation rate $(\varepsilon)$ and specific dissipation rate $(\omega)$; while the second order accurate scheme has been used for the Poisson-like pressure equation. For the stream wise velocity, inlet boundary conditions have been generated in C-language. For this, the interface of User Defined Function (UDF) of Fluent has been used and then linked with the solver. The initialization of solution process has been done by using random values of the velocity components, $k-\omega$ and $k-\varepsilon$. Residuals of these parameters have been monitored at each iteration. However, the gradual drops of residuals strongly indicate for the accurate and stable solutions. Finally, iteration process is terminated when the residuals are leveled off at $10^{-6}$ and the final converged solutions are achieved.

\subsection{Grid Independency Test}

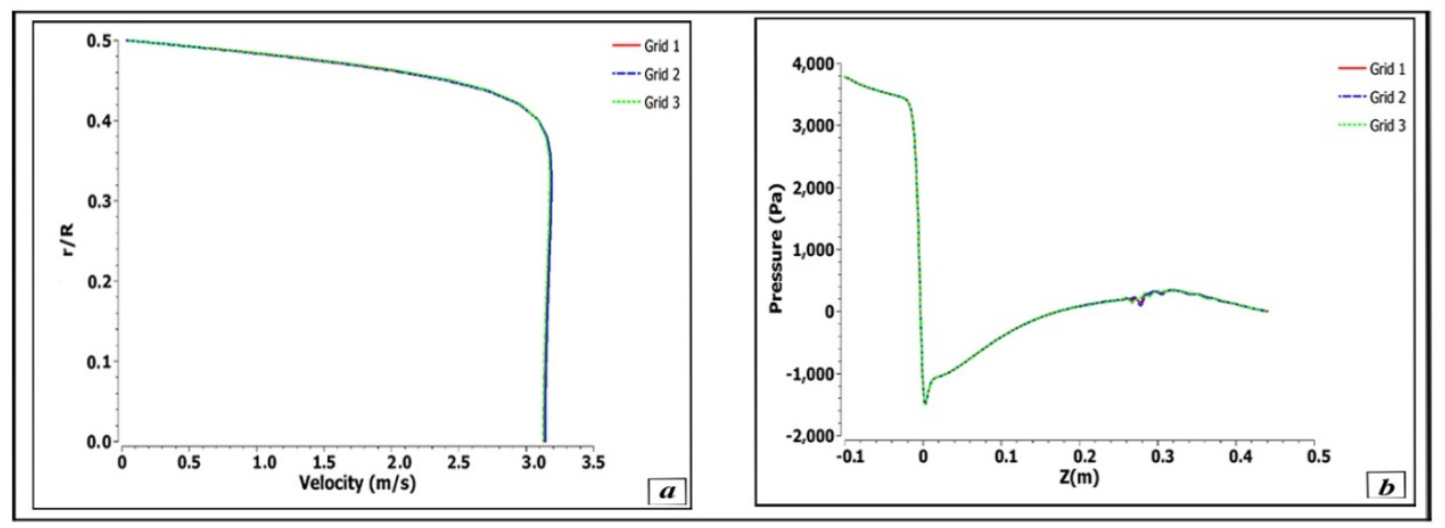

Fig. 2.2. Grid independency test for three different grids: (a) axial velocity at the throat of the stenosis and (b) centerline pressure along the artery.

In the Fig. 2.2(a), we can see that the velocity along the axis of the artery remains almost same for three different grids shown in the table 2.1 .

Table 2.1: Three different grids

\begin{tabular}{ll}
\hline Grid & $\begin{array}{l}\text { Number of control } \\
\text { volume }\end{array}$ \\
\hline Grid 1 & 127450 \\
Grid 2 & 137550 \\
Grid 3 & 147500 \\
\hline
\end{tabular}

The centerline pressure is also similar for different grids which are shown in Fig. 2.2(b). Initially, Grid 2 is increased about $8 \%$ in terms of control volumes than Grid 1 . While Grid 3 has about 16\% increased control volumes than Grid 1. But, there is no significant change in corresponding value for different grids. Finally, we have discretized the computational domain using Grid 3. 


\section{Results and Discussion}
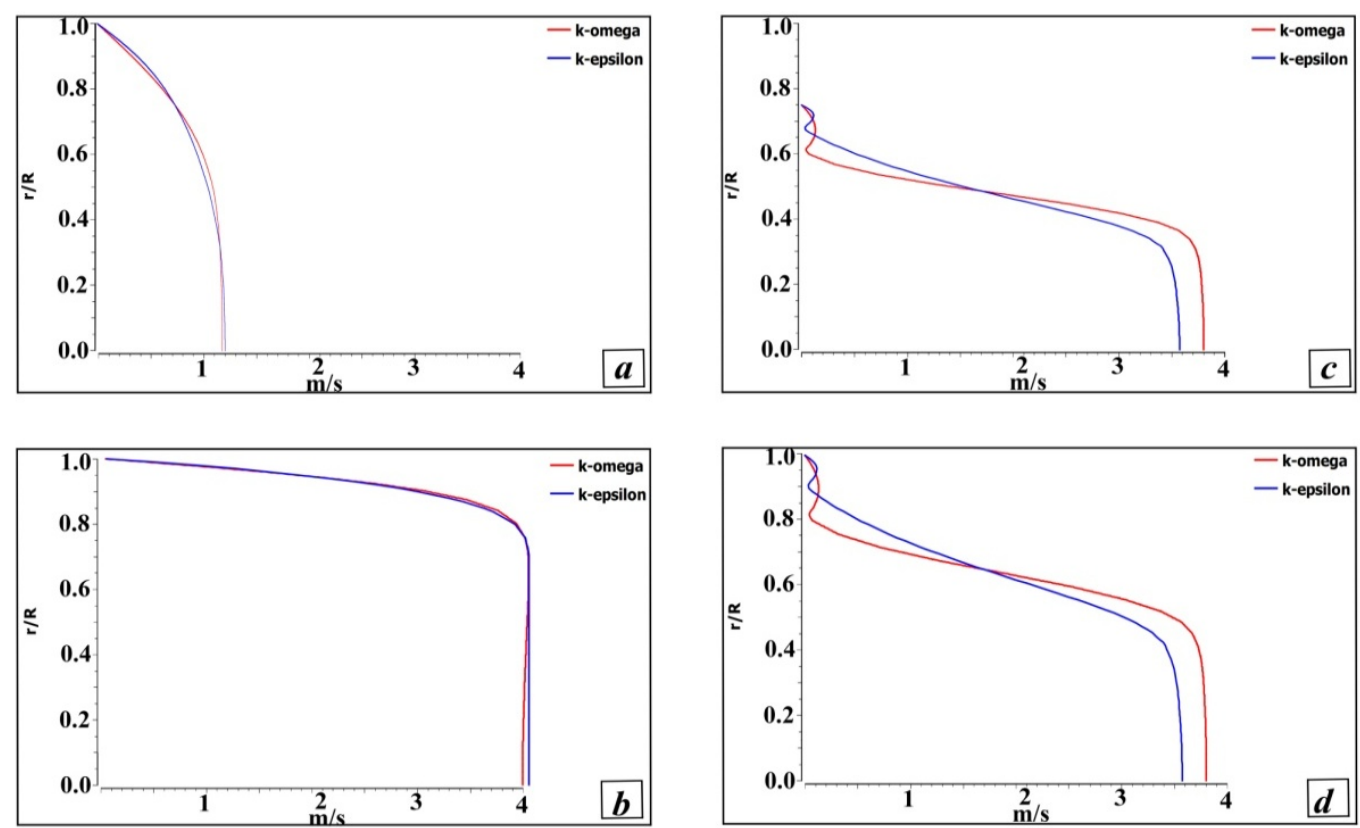

Fig. 3.1. Velocity variations at the different axial positions (a) $-1 \mathrm{D}$,(b) $0 \mathrm{D}$, (c) $0.5 \mathrm{D}$ and (d) $1 \mathrm{D}$ at $\mathrm{Re}=2000$.

The axial velocity distributions by using both $k-\omega$ and $k-\varepsilon$ turbulence models are plotted in Fig. 3.1 and 3.2. at different axial locations such as $-1 \mathrm{D}, 0 \mathrm{D}, 0.5 \mathrm{D}, 1 \mathrm{D}, 2 \mathrm{D}, 3 \mathrm{D}, 4 \mathrm{D}$ and $5 \mathrm{D}$. The velocity differences at different positions were compared by using the results obtained from the simulations from both of the models (i.e. $k-\omega$ and $k-\varepsilon$ models). Findings show that, the velocity at $-1 \mathrm{D}$ and $0 \mathrm{D}$ position using $k-\omega$ and $k-\varepsilon$ models almost collapse with each other. It is also important to note that, the velocity does not vary significantly in the simulations from two different models at those positions. However, at $0.5 \mathrm{D}$ and $1 \mathrm{D}$ positions, though velocity profiles show similar pattern, the velocity from $k$ - $\omega$ and $k-\varepsilon$ models vary significantly. At the $2 \mathrm{D}, 3 \mathrm{D}, 4 \mathrm{D}$ and $5 \mathrm{D}$ positions, the velocity profiles using $k$ - $\omega$ model agree well with the experimental data obtained by Ahmed and Giddens [18], however $k-\varepsilon$ model loses its stability at the poststenotic region. This loss of stability in the velocity profiles in the $k-\varepsilon$ model differs significantly from the results from the $k$ - $\omega$ model. Thus, these results enable us to conclude that the results obtained by using $k-\omega$ model are better than that of $k-\varepsilon$ model in terms of velocity. Existing studies also suggest that, the performance of $k-\omega$ model is better than that of $k-\varepsilon$ model in terms of velocity. For example, velocity from the $k$ - $\omega$ turbulence model shows good agreement with the experimental data [18]. 

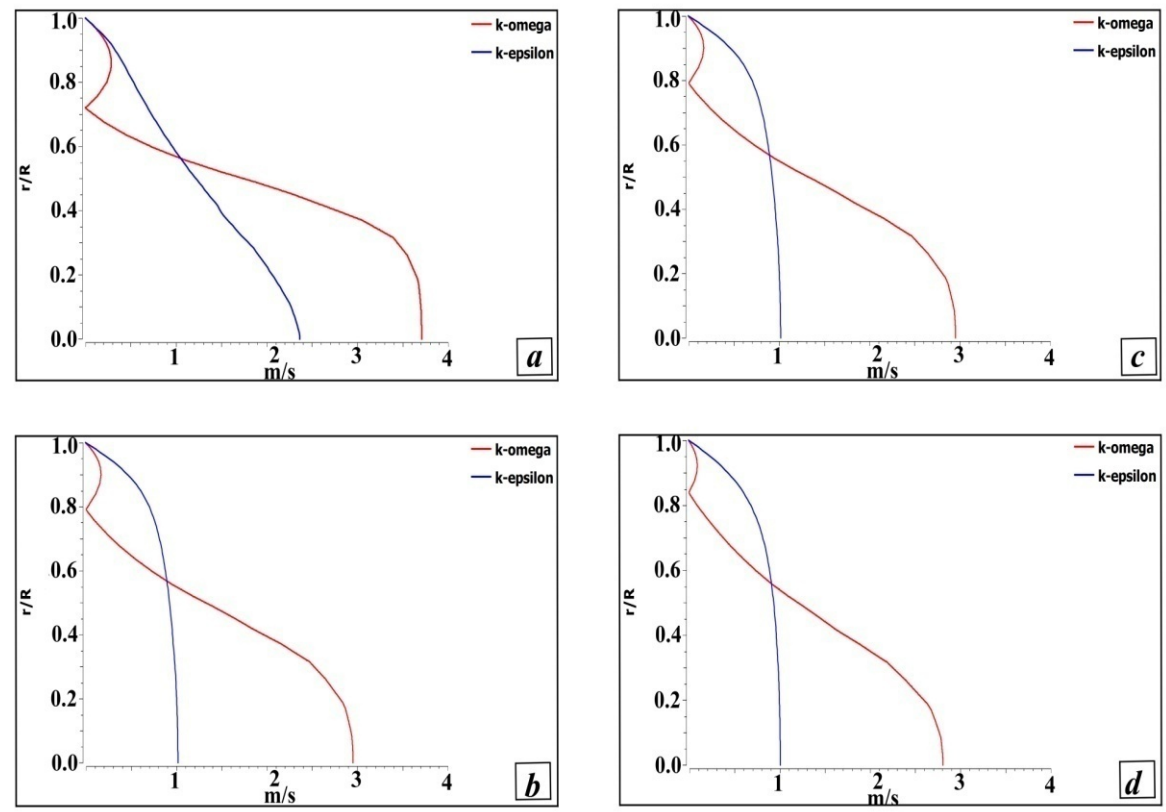

Fig. 3.2. Velocity variations at the different axial positions (a) 2D, (b) 3D, (c) 4D and (d) 5D.

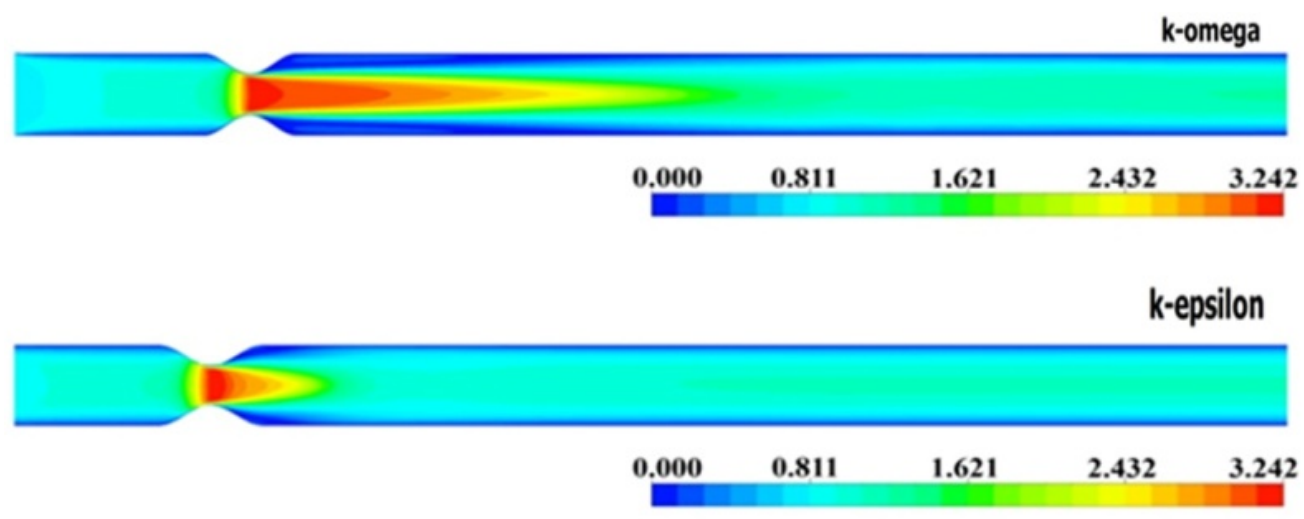

Fig. 3.3. Velocity contour plots at XY-plane for $k-\omega$ and $k-\varepsilon$ respectively.

The velocity contour plots for the $k-\omega$ and $k-\varepsilon$ models are shown in Fig. 3.3 where velocity contours are plotted towards the distance of the artery. Variation in streamwise velocities at different positions is also compared for the $k$ - $\omega$ and $k-\varepsilon$ models. Findings show that, the intensity of streamwise velocity using $k-\omega$ model is higher than that of $k-\varepsilon$ model which suggest that the $k-\omega$ model can capture the large scale velocity variation comparing to the $k-\varepsilon$ model. With the increase in the distance towards the downstream, we observed the variation in stream wise velocity between the $k$ - $\omega$ and $k-\varepsilon$ and found that turbulent models are significant.

The stream wise velocity profiles for two different models are shown in Fig. 3.4. From the results of two models it has been observed that, the $k-\omega$ model can capture the large scale and even minor change in the velocity. However, the $k-\varepsilon$ model cannot do that. For example, the $k-\omega$ model can capture the large scale variation in streamwise velocity while in the $k-\varepsilon$ model this variation is observed for the small region. 


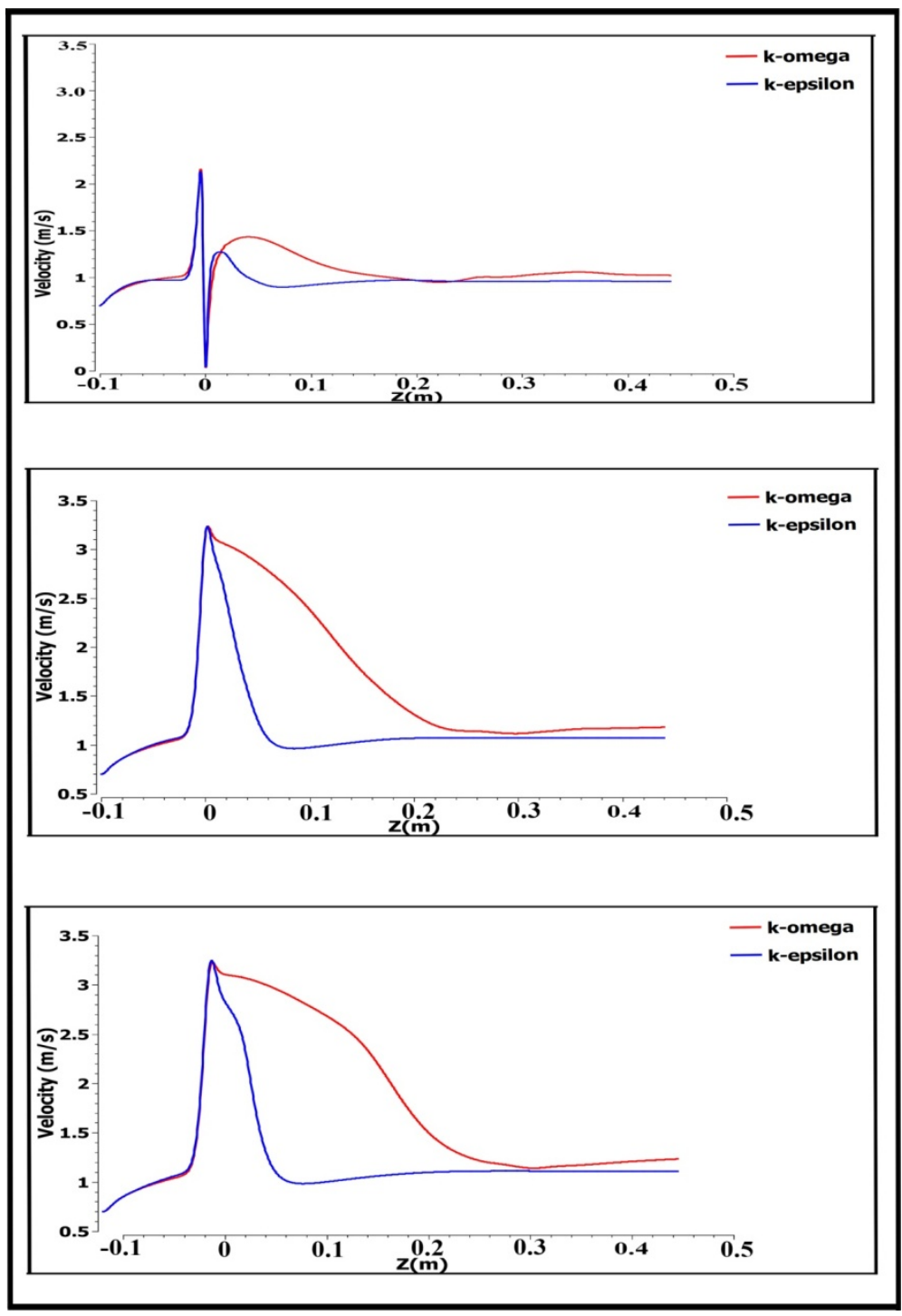

Fig. 3.4. Streamwise velocity profiles of $k-\omega$ and $k-\varepsilon$ for (a) $r / R=0.4$, (b) $r / R=0.2$ and (c) $r / R=0.0$ positions.

The variation in radial velocity at different positions is also compared for the $k-\omega$ and $k-\varepsilon$ models and their results are shown in Fig. 3.5 where velocity is plotted along the distance of the artery. The results obtained from the observed data show that, the streamwise velocity, far away from the centerline position, shows similar pattern both in $k-\omega$ and $k-\varepsilon$ models. Interestingly, centerline radial velocities vary significantly at downstream region of the artery which is shown in Fig. 3.5(c). This reveals that the $k-\omega$ model is able to capture the small variation in radial velocity in the downstream region. On the other hand, $k-\varepsilon$ model is not able to capture such types of peak. This indicates that the $k-\omega$ model is much more efficient than the $k-\varepsilon$ model. 


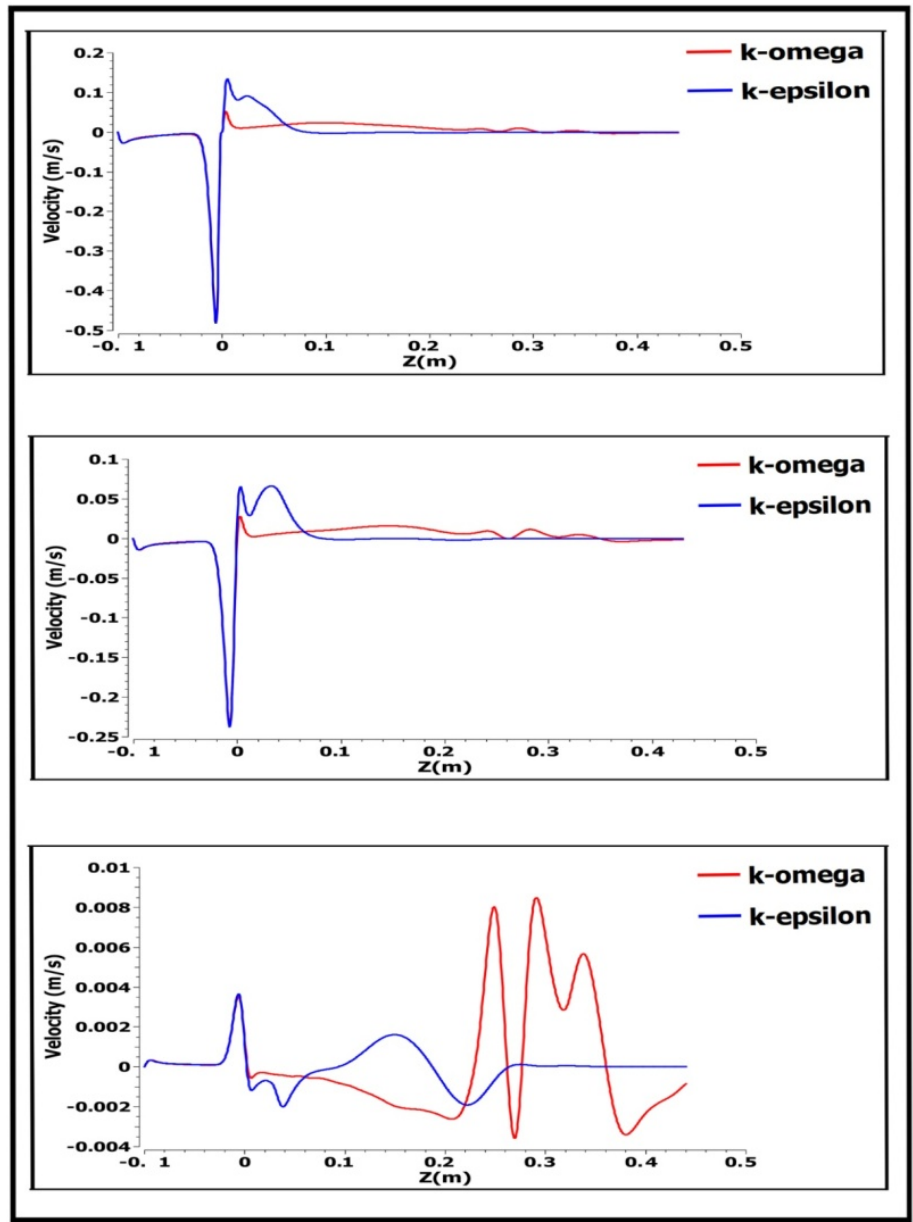

Fig. 3.5. Variation in radial velocity of $k-\omega$ and $k-\varepsilon$ models for (a) $r / R=0.4$, (b) $r / R=0.2$ and (c) $r / R=0.0$ positions.

Radial velocity contour plot shows that the $k$ - $\omega$ model can capture the large scale and even minor change in the velocity. However, the $k-\varepsilon$ model cannot do that properly which is one of the major deficiencies of this model. We found that, at the upstream region to near the throat of the stenosis both of $k-\omega$ and $k-\varepsilon$ models have similar patterns of velocities.

The tangential velocity profiles for two existing models at three different positions are shown in Fig. 3.6.The stream wise tangential velocity in $k-\omega$ model and $k-\varepsilon$ model showed significant differences; interestingly we did not find any difference for tangential velocity at the upstream region. However, at the downstream region the tangential velocity from $k-\omega$ and $k-\varepsilon$ models vary significantly. Similar to radial velocity, $k-\omega$ model is also able to capture even small variations of tangential velocities effectively. Tangential velocity contour plot shows that the $k-\omega$ model can capture the large scale and minor change in the velocity. However, the $k-\varepsilon$ model cannot do that properly. 

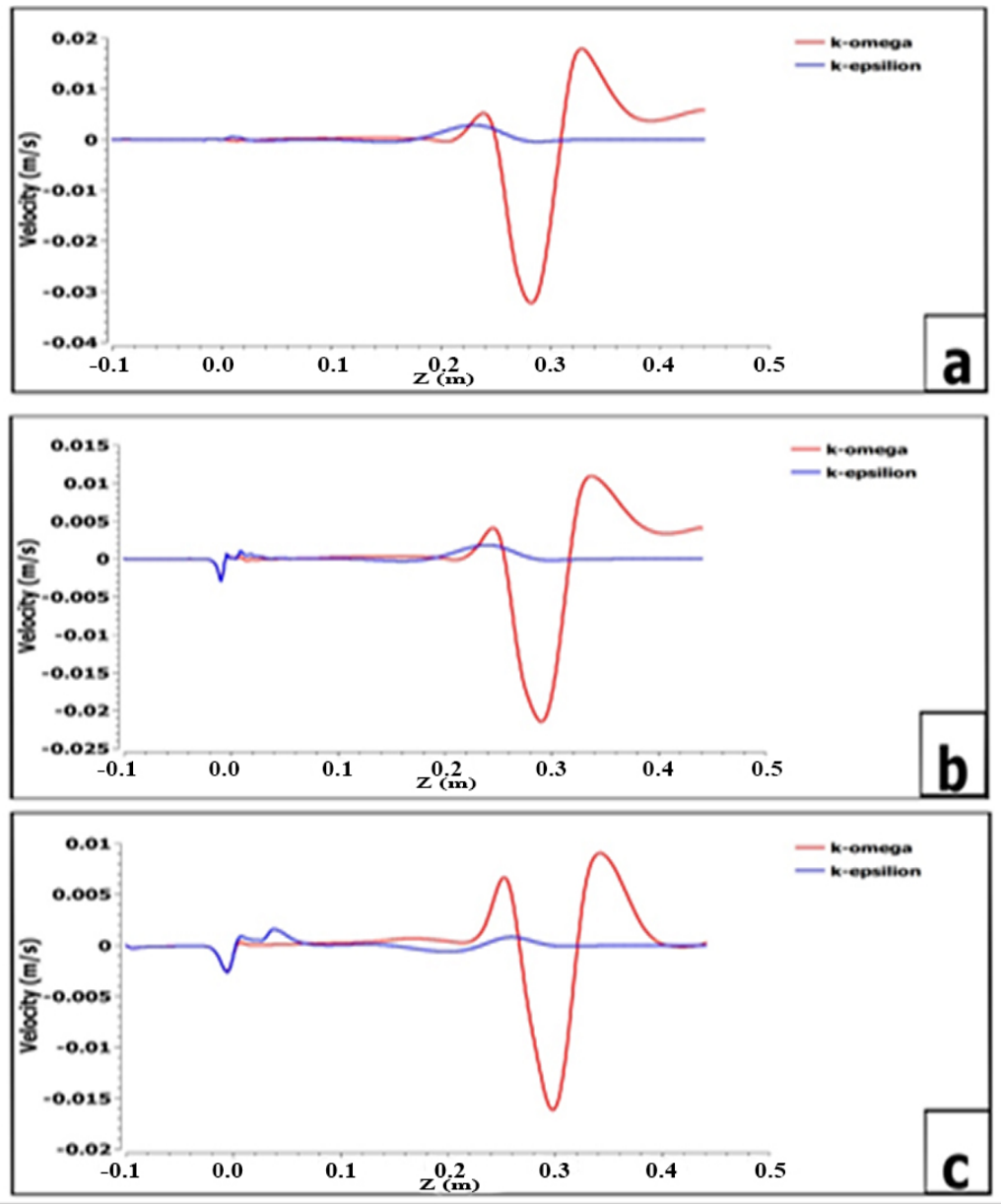

Fig. 3.6. Variation in tangential velocity of $k-\omega$ and $k-\varepsilon$ models for (a) $r / R=0.4$, (b) $r / R=0.2$ and (c) $r / R=0.0$ positions.

The turbulence kinetic energy (TKE) at three different radial positions for models $k-\omega$ and $k-\varepsilon$ are shown in Fig. 3.7. The $k-\omega$ and $k-\varepsilon$ models show that at the upstream region the turbulence kinetic energy vary significantly. But the turbulence kinetic energy remains almost same in both $k-\omega$ and $k-\varepsilon$ models at the downstream region. The contour plots of TKE for two models are also shown in Fig. 3.10. The results of contour plots illustrate that $k$ - $\omega$ model can predict even small variations of TKE at the post-stenotic zone but $k-\varepsilon$ cannot predict TKE at that zone properly. 


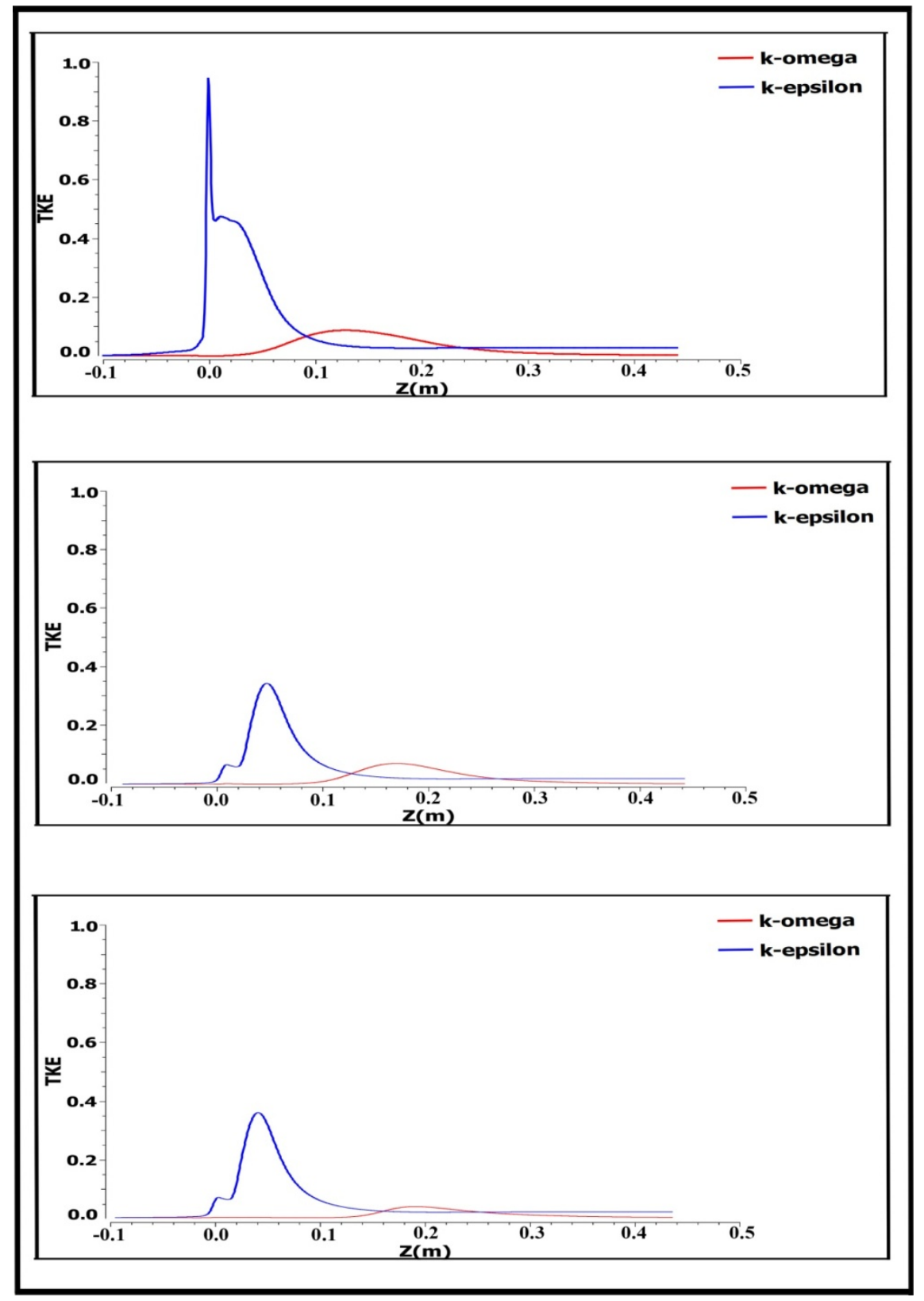

Fig. 3.7. Variation in turbulent kinetic energy (TKE) of $k-\omega$ and $k-\varepsilon$ models for (a) $r / R=0.4$, (b) $r / R=0.2$ and (c) $\mathrm{r} / \mathrm{R}=0.0$ positions. 


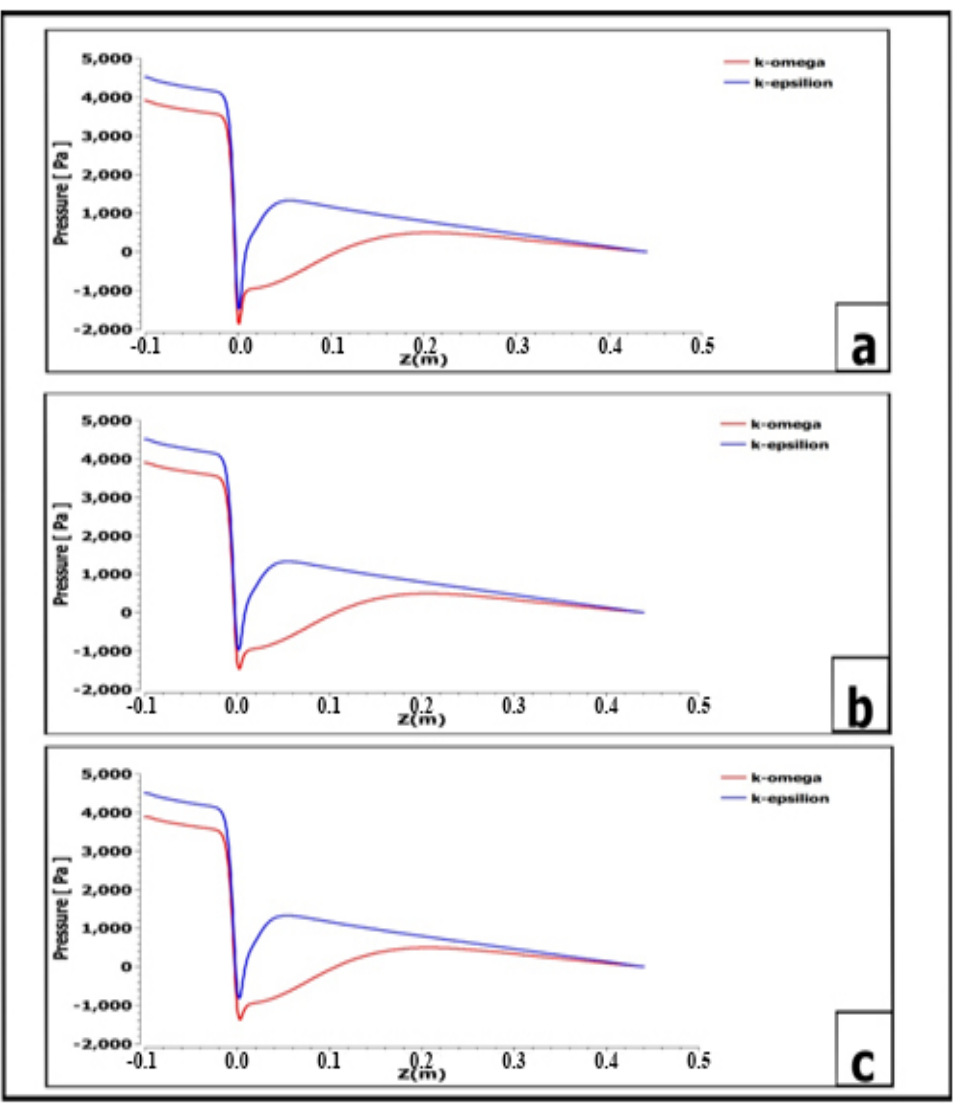

Fig. 3.8. Variation in pressure of $k-\omega$ and $k-\varepsilon$ model at $r / R=0.4$, (b) $r / R=0.2$ and (c) $r / R=0.0$ positions.

The pressure distributions from $k-\omega$ and $k-\varepsilon$ models are shown in Fig. 3.8. For both models, pressure drop at the throat of the stenosis and $k-\omega$ model can predict more pressure drop compared to $k-\varepsilon$ model and this intensity of pressure drop for $k-\omega$ have been found up to the $15 \mathrm{D}$ position. At the end of the downstream region, the pressure remains almost same of the $k-\omega$ and $k-\varepsilon$ models. It is important to note that the pressure pattern found from the simulation in $k-\omega$ model is more realistic than that of $k-\varepsilon$ model simulations.

The wall shear stress for two turbulent models is given in Fig. 3.9. At the upstream region the wall shear stress remains similar in $k-\omega$ and $k-\varepsilon$ models. In the downstream region, there is a slight difference observed between $k$ - $\omega$ and $k-\varepsilon$ models. The $k-\omega$ model can capture even minor change in the wall shear stress in the downstream region while $k-\varepsilon$ model cannot capture those changes. However, at the throat, the $k-\varepsilon$ model can capture the variation in the WSS more efficiently than $k$ - $\omega$ model. Contour plot for TKE for $k$ - $\omega$ model and $k$ - $\varepsilon$ model are shown in Fig. 3.10 and eddy viscosity is shown in Fig. 3.11. After the throat, the $k-\omega$ model can capture the variation in TKE, while the results from the $k-\varepsilon$ model show that there are no major changes in TKE after the stenosis. Similar types of results were also observed for the eddy viscosity. After the stenosis the $k$ - $\omega$ model can capture the major gradients in eddy viscosity. 


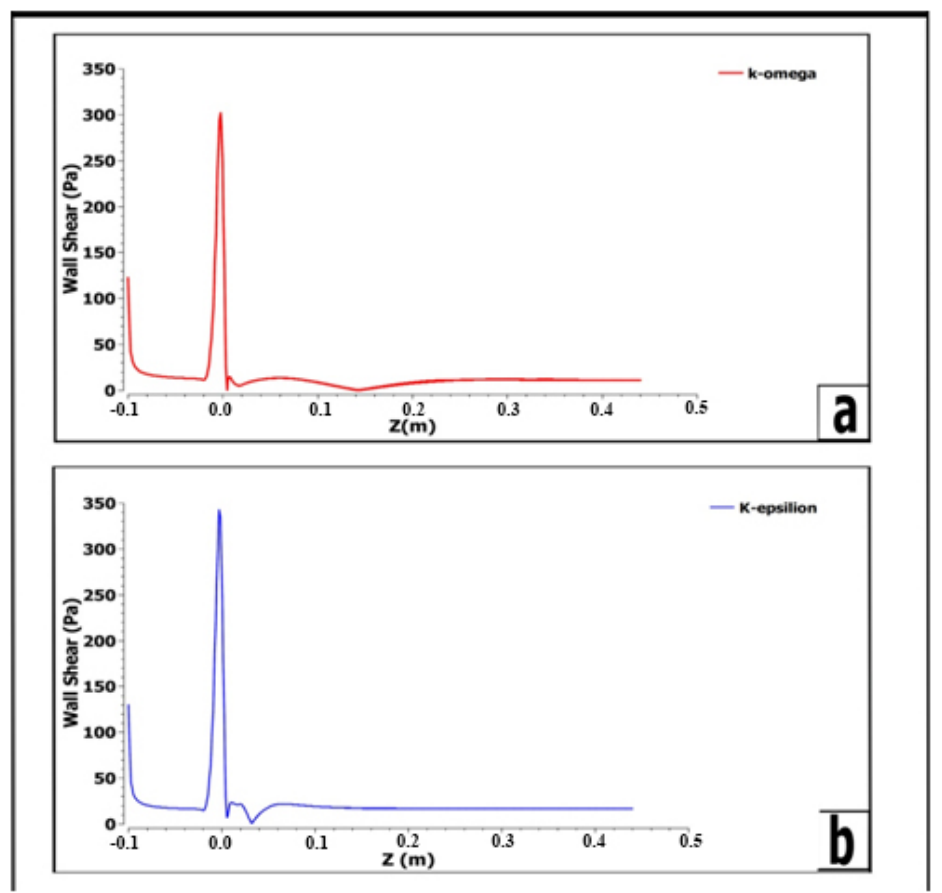

Fig. 3.9. Variation in wall shear stress at XY-plane for $k-\omega$ and $k-\varepsilon$ model.

On the other hand, in the $k-\varepsilon$ model no significant variation in the eddy viscosity is found after the stenosis. These findings reveal that $k-\omega$ turbulent model can perform better than that of the $k-\varepsilon$ model.

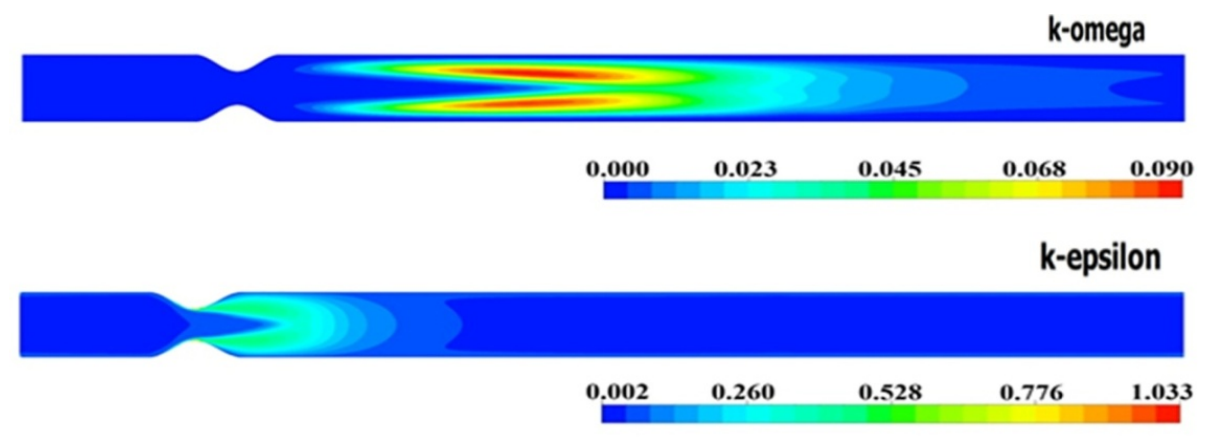

Fig. 3.10. Variation in contour plot of TKE at XY-plane for $k-\omega$ and $k-\varepsilon$ models.
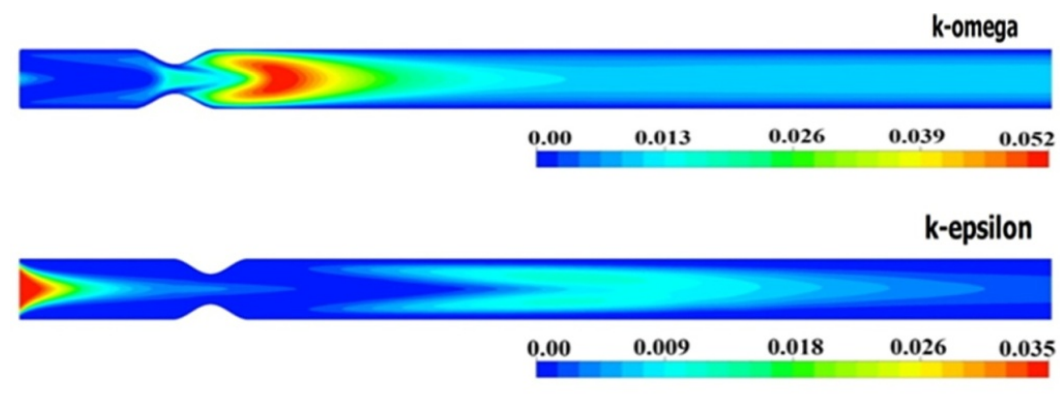

Fig. 3.11. Variation in contour plot of eddy viscosity at XY-plane for $k-\omega$ and $k-\varepsilon$ models.

The two-equation $k$ - $\omega$ turbulence model solves transport equations for the turbulent kinetic energy $(k)$ and the specific dissipation rate $(\omega)$, which is the turbulent dissipation rate $(\varepsilon)$ per unit turbulent kinetic energy. Menter [19] modified two-equation $k-\omega$ turbulence models for aerodynamic flows. This new model is also identical to the Jones- 
Lauder model for free shear layers. Goldberg and Apsley [20] presented $k$ - $\varepsilon$ turbulence model for low Reynolds number based on the following three attributes: (1) it involves neither explicit wall distance nor normal-to-wall directionality, except to the first grid point off walls; (2) it enforces time scale reliability; (3) it involves a simple wall boundary condition for $k-\omega$. Kalita et al. [21] used the $\mathrm{k}-\epsilon-\mathrm{t}^{\prime} \quad 2-\gamma$ model to present the computation of the turbulent plane plume. Song et al. [22] investigated turbulence models in a CFD model of a blood pump. They employed CFD simulations of blood flow in a centrifugal left ventricular assist device. For the initial numerical calculation, a $k-\varepsilon$ model with near wall functions was used. Local grids with special distribution were used to improve simulation. In their study, a comparison was made between $k-\omega$ model and experimental measurements of the flow field found by particle image velocimetry. It showed better agreement than $k-\varepsilon$ model does mostly in the near wall regions. The Spalart-Allmaras and the Menter (1994) SST $k-\omega$ turbulence models provide the peculiar characteristic that, for fully turbulent computations, a transition region sometimes occur whose extent changes with grid density. Two equation $k-\omega$ models and the SST $k-\omega$ model show a powerful sensitivity to numerical resolution near the area where turbulence initiation occurs [23]. Zhang et al. [24] investigated a 3D non-linear $k-\varepsilon$ turbulent model for guessing flow and mass transport in channel with vegetation. This 3D numerical model is used for calculating flow in non-orthogonal curvilinear coordinates to compute the complex boundary channel. However results obtained from this model didn't fit too much with the experimental data. Sonntag et al. [25] merged computational and experimental approach to improve the assessment of mitral regurgitation by echocardiography. They concluded that the performance of $k-\omega$ model is more efficient than $k-\varepsilon$ model to mimic the experimental data.

For this study experimental data are compared with the simulation results from $k-\omega$ and $k-\varepsilon$ model. The comparison is shown in Fig. 3.12. Comparison shows that, simulation results from $k$ - $\omega$ model has comparatively better match with experimental data than that of $k-\varepsilon$ model. This suggests that, performance of $k-\omega$ model is better than that of $k-\varepsilon$ model.

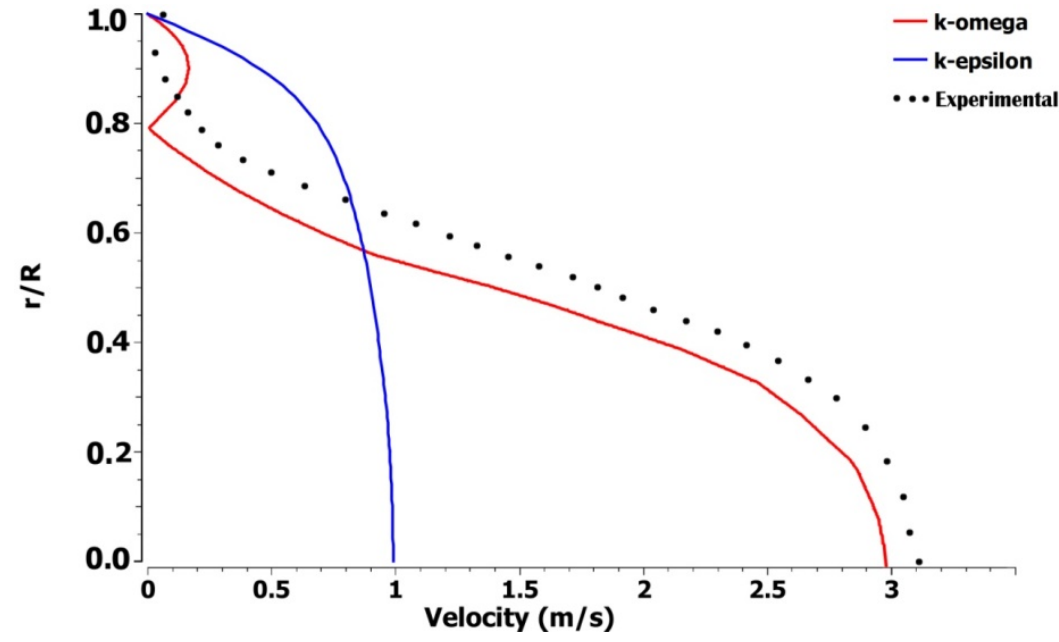

Fig. 3.12. Velocity comparison of $k-\omega$ and $k-\varepsilon$ with experimental data of Ahmed and Giddense at 4D axial position.

\section{Conclusions}

Computational fluid dynamics (CFD) is a handy tool for predicting turbulence in biomedical and aerodynamic applications. Hence, the selection of appropriate turbulence model is a must to obtain correct predictions. Reynoldsaveraged Navier-Stokes (RANS) equations have become the popular and wide spread standard for modeling turbulent flows. These models try to close the turbulence equations using viscosity terms. In the current study, we employed $k-\omega$ model and $k-\varepsilon$ model to simulate the blood flow through stenosed artery. The purpose of the study was to find out the performance of these models. However, from the observed data it was found that, the flow parameters obtained from the $k-\varepsilon$ turbulent model has lack of fits with the experimental data. On the other hand, $k-\omega$ turbulent model can capture large scale gradient in different parameters of blood flow and has a good agreement with the experimental data. This study concludes that $k-\omega$ model has the better performance compared to $k$ - $\varepsilon$ model in predicting the behavior of blood flow and it can be chosen as a better modeling option in the fluid dynamics specially in bio-fluid field. 


\section{Acknowledgements}

The first author is grateful to the Ministry of Science and Technology, Government of Bangladesh for NST fellowship to carry out this study.

\section{Funding}

This research is partially funded by SUST research center which is thankfully acknowledged.

\section{References}

[1] Cassanova, R.A. and D.P. Giddens, Disorder distal to modeled stenoses in steady and pulsatile flow. Journal of Biomechanics,11(10): 441-453 (1978).

[2] Boughner, D.R. and M.R. Roach, Effect of Low Frequency Vibration on the Arterial Wall. Circulation Research,29(2): 136-144 (1971).

[3] Ghalichi, F., et al., Low Reynolds number turbulence modeling of blood flow in arterial stenoses. Biorheology,35(4): 281-294 (1998).

[4] Ahmed, S.A. and D.P. Giddens, Velocity measurements in steady flow through axisymmetric stenoses at moderate Reynolds numbers. Journal of Biomechanics, 16(7): 505-516 (1983).

[5] Clark, C., The fluid mechanics of aortic stenosis-I. Theory and steady flow experiments. Journal of Biomechanics, 9(8): 521-528 (1976).

[6] Giddens, D.P., R.F. Mabon, and R.A. Cassanova, Measurements of disordered flows distal to subtotal vascular stenoses in the thoracic aortas of dogs. Circ Res,39(1): 112-9 (1976).

[7] Strandness, D.E., Vascular diseases: current research and clinical applications: Grune \& Stratton, Incorporated (1987).

[8] Kabir, M., M.F. Alam, and M. Ashraf Uddin, A Numerical Study on the Effects of Reynolds Number on Blood Flow with Spiral Velocity Through Regular Arterial Stenosis. Chiang Mai Journal of Science,45(x): 113 (2018).

[9] Kabir, M., M.F. Alam, and M. Uddin, Numerical Simulation of pulsatile blood flow: A study with normal artery, and arteries with single and multiple stenosis. Egyptian Jounal of Basic and Applied Sciences, (Accepted).

[10] Lee, J.-S. and Y.-C. Fung, Flow in Locally Constricted Tubes at Low Reynolds Numbers. Journal of Applied Mechanics,37(1): 9-16 (1970).

[11] Daly, B.J., A numerical study of pulsatile flow through stenosed canine femoral arteries. Journal of Biomechanics,9(7): 465-475 (1976).

[12] Deshpande, M.D., D.P. Giddens, and R.F. Mabon, Steady laminar flow through modelled vascular stenoses. Journal of Biomechanics,9(4): 165-174 (1976).

[13] Deshrande, D.M., Steady laminar and turbulent flow through vascular stenosis models, (1977).

[14] Ku, D.N., BLOOD FLOW IN ARTERIES. Annual Review of Fluid Mechanics, 29(1): 399-434 (1997).

[15] Lee, T.S., W. Liao, and H.T. Low, Numerical simulation of turbulent flow through series stenoses. International Journal for Numerical Methods in Fluids, 42(7): 717-740 (2003).

[16] Smith, F.T., On entry-flow effects in bifurcating, blocked or constricted tubes. Journal of Fluid Mechanics, 78(4): 709-736 (2006).

[17] Patankar, S.V. and D.B. Spalding, A calculation procedure for heat, mass and momentum transfer in threedimensional parabolic flows. International Journal of Heat and Mass Transfer,15(10): 1787-1806 (1972).

[18] Ahmed, S.A. and D.P. Giddens, Pulsatile poststenotic flow studies with laser Doppler anemometry. Journal of Biomechanics, 17(9): 695-705 (1984).

[19] Menter, F., Improved two-equation k-omega turbulence models for aerodynamic flows, 93 (1992).

[20] Goldberg, U. and D. Apsley, A wall-distance-free low Re k - $\epsilon$ turbulence model. Computer Methods in Applied Mechanics and Engineering, 145(3): 227-238 (1997).

[21] Kalita, K., A. Dewan, and A.K. Dass, Computation of the turbulent plane plume using the $\mathrm{k}-\epsilon-\mathrm{t}^{\prime} 2-\gamma$ model. Applied Mathematical Modelling, 24(11): 815-826 (2000).

[22] Song, X., et al., Studies of Turbulence Models in a Computational Fluid Dynamics Model of a Blood Pump .Artificial Organs, 27(10): 935-937 (2003).

[23] Rumsey, C.L., Apparent transition behavior of widely-used turbulence models. International Journal of Heat and Fluid Flow, 28(6): 1460-1471 (2007).

[24] Zhang, M.-L., C.W. Li, and Y.-M. Shen, A 3D non-linear k- $\varepsilon$ turbulent model for prediction of flow and mass transport in channel with vegetation. Applied Mathematical Modelling, 34(4): 1021-1031 (2010).

[25] Sonntag, S.J., et al., Combined Computational and Experimental Approach to Improve the Assessment of Mitral Regurgitation by Echocardiography. Annals of Biomedical Engineering, 42(5): 971-985 (2014). 